\title{
Self-repairing Adaptive PID Control for Plants with Sensor Failures
}

\author{
Masanori Takahashi \\ Department of Electrical Engineering and Computer Science, Tokai University \\ 9-1-1 Toroku, Higashi-ku, Kumamoto, 862-8652, Japan \\ E-mail: masataka@ktmail.tokai-u.jp
}

\begin{abstract}
This paper presents a new design method for a self-repairing adaptive PID control system. The control system has the adaptive adjusting mechanisms for the PID gains, and also can detect the sensor failure by self-test using the integrator in the PID controller. Hence, for plants with unknown parameters, self-repairing control can be successfully attained. Furthermore, in this paper, the control performances are theoretically analyzed.
\end{abstract}

Keywords: PID control, Adaptive control, Fault-tolerant control, Sensor failure.

\section{Introduction}

Against sensor failure, several types of self-repairing control systems (SRCS) have been developed in the previous works. The SRCS can detect failure by selftesting using the internal signals, and automatically replace the failed sensor with the healthy backup to maintain its stability. They belong to active fault-tolerant control (AFTC) based on dynamic redundancy ${ }^{1}$. The main difference (feature point) from the conventional AFTC is that the fault detector is quite simple, and its structure does not depend on the mathematical model of the plant. ${ }^{2,3,4}$ For example, the integrator in the PID controller can be utilized as fault detector for the $\mathrm{SRCS}^{5}$.

However, in most existing SRCSs, roughly estimated parameters of plants are required to construct the controller. This paper presents a new design method for a self-repairing adaptive PID control system. The control system has the adaptive adjusting mechanisms for the PID gains, and also can detect the sensor failure by self- test using the integrator in the PID controller. Thus, no $a$ priori information about the plant is needed to attain the SRC. The proposed SRCS is expected to be widely utilized as fault-tolerant PID control.

Furthermore, in this paper, the control stability, failure detection and sensor-recovery are theoretically analyzed. In addition, the numerical simulation is explored to confirm the effectiveness of the proposed adaptive SRCS.

Throughout this paper, with $x \in \mathbb{R}$, we define the "sgn" function by

$$
\operatorname{sgn}[x]=\left\{\begin{array}{cc}
1 & (x \geq 0) \\
-1 & (x<0)
\end{array}\right.
$$

Notice that this is slightly different from the ordinary one.

\section{Problem Statement}

Consider the following linear time invariant system:

$$
\begin{aligned}
& \dot{y}=a y+b u+\boldsymbol{h}^{T} \boldsymbol{z} \\
& \dot{\mathbf{z}}=\boldsymbol{F} \mathbf{z}+\boldsymbol{g} y
\end{aligned}
$$


where $y \in \mathbb{R}$ is the actual output, $u \in \mathbb{R}$ is the input, and $\mathbf{z} \in \mathbb{R}^{n-1}$ is the state of the plant. Here, assume that the plant is minimum-phase, that is, all eigenvalues of $\boldsymbol{F} \in \mathbb{R}^{(n-1) \times(n-1)}$ lie in $\mathbb{C}^{-}$.

For measurement of the output $y$, the two sensors are exploited; one is the primary sensor \#1 and the other is the backup sensor \#2 for occasion of failure. Then, the measured feedback signal can be represented as

$$
y_{S}(t)= \begin{cases}y_{1}(t) & \left(t \leq t_{D}\right) \\ y_{2}(t) & \left(t>t_{D}\right)\end{cases}
$$

where $y_{i} \in \mathbb{R}, i=1,2$ is the output of the sensor $\# i$, and $t_{D} \in \mathbb{R}$ is the detection time whose details will be determined later. If the sensors are healthy, then the measured signals are equivalent to the actual output, that is, we have $y_{i}=y$. Unfortunately, the primary sensor fails in the following way.

$$
y_{1}(t)=\varphi, t \geq t_{F}
$$

where $t_{F} \in \mathbb{R}^{+}$is the unknown failure time, and $\varphi \in \mathbb{R}$ is the unknown stuck value.

The problem to be considered here, is to construct the active fault-tolerant PID control system for plant with unknown parameters and sensor failures (3), that can automatically replace the failed sensor with the backup to maintain the control stability.

\section{Basin Design of the Control System}

First of all, the adaptive PID controller is designed by

$$
u=k_{P}\left(-y_{S}+v\right)-k_{D} \dot{y}_{S}
$$

where $v \in \mathbb{R}$ is the output of the integrator:

$$
\dot{v}=-k_{I} y_{S}-\tau \operatorname{sgn}\left[y_{S}\right]
$$

with a constant $\tau \in \mathbb{R}^{+}$, which is introduced for detection of failure. The fault detection using the signal $v$ will be discussed in the next section.

In the above adaptive controller, the PID gains, $k_{P}: \mathbb{R}^{+} \rightarrow$ $\mathbb{R}^{+}, k_{I}: \mathbb{R}^{+} \rightarrow \mathbb{R}$ and $k_{D}: \mathbb{R}^{+} \rightarrow \mathbb{R}$ are adaptively tuned as follows.

$$
\begin{aligned}
& \dot{k}_{P}=-\sigma k_{P}+\gamma\left(-y_{S}+v\right)^{2} \\
& \dot{k}_{I}=-\sigma k_{I}-\gamma y_{S}\left(y_{S}-2 v\right) \\
& \dot{k}_{D}=-\sigma k_{D}-\delta \gamma \dot{y}_{S}\left(-y_{S}+v\right)
\end{aligned}
$$

where $\sigma \in \mathbb{R}^{+}$is an any small constant, and $\gamma \in \mathbb{R}^{+}$is a positive constant. Also, a small constant $\delta \in \mathbb{R}^{+}$is introduced as a scaling factor. These are given by designers.

Here, we shall analyze the control stability on the time period $\left[0, t_{F}\right)$ where the sensor is healthy, i.e., $y_{S}=y$. Now, define the augmented signal $\varepsilon: \mathbb{R}^{+} \rightarrow \mathbb{R}$ by

$$
\varepsilon:=-y_{S}+v
$$

Then, the signals, $\varepsilon, \mathbf{z}, v$ obey the following equations.

$$
\begin{aligned}
\dot{\varepsilon}= & \left(\frac{1}{1+b k_{D}^{*}}\right)\left\{-\left(b k_{P}^{*}-a-k_{I}^{*}+b k_{D}^{*} k_{I}^{*}\right) \varepsilon+b \Delta_{P} \varepsilon\right. \\
& -\Delta_{I}(\varepsilon-v)-\left(k_{I}^{*}+a-b k_{D}^{*} k_{I}^{*}\right) v-b \Delta_{D} \dot{y} \\
& \left.-\boldsymbol{h}^{T} \boldsymbol{z}-\left(1-b k_{D}^{*}\right) \tau \operatorname{sgn}[y]\right\} \\
\dot{\mathbf{z}}= & \boldsymbol{F} \mathbf{z}-\boldsymbol{g} \varepsilon+\boldsymbol{g} v \\
\dot{v}= & -k_{I}^{*} v+k_{I}^{*} \varepsilon-\Delta_{I}(\varepsilon-v)-\tau \operatorname{sgn}[y]
\end{aligned}
$$

where $\Delta_{P}:=k_{P}^{*}-k_{P}, \Delta_{I}:=k_{I}^{*}-k_{I}$ and $\Delta_{D}:=k_{D}^{*}-$ $k_{D}$, and $k_{P}^{*} \in \mathbb{R}^{+}, k_{I}^{*} \in \mathbb{R}^{+}$and $k_{D}^{*} \in \mathbb{R}^{+}$are the ideal gains for PID control.

Consider the positive definite function $S: \mathbb{R}^{+} \rightarrow \mathbb{R}^{+}$:

$S=$

$\frac{1}{2}\left\{\left(1+b k_{D}^{*}\right) \varepsilon^{2}+\mathbf{z}^{T} \boldsymbol{P} \mathbf{z}+v^{2}+\frac{b}{\gamma} \Delta_{P}^{2}+\frac{1}{\gamma} \Delta_{I}^{2}+\frac{b}{\delta \gamma} \Delta_{D}^{2}\right\}$

where $\boldsymbol{P} \in \mathbb{R}^{(n-1) \times(n-1)}$ is the positive definite matrix which satisfies $\boldsymbol{F}^{T} \boldsymbol{P}+\boldsymbol{P} \boldsymbol{F}=-2 \boldsymbol{Q}$ for any positive definite $\boldsymbol{Q} \in \mathbb{R}^{(n-1) \times(n-1)}$.

Taking the time derivative of $S$, gives

$$
\begin{aligned}
\dot{S}= & -\left(b k_{P}^{*}-a-k_{I}^{*}+b k_{D}^{*} k_{I}^{*}\right) \varepsilon^{2} \\
& +b \Delta_{P} \varepsilon^{2}-\Delta_{I}(\varepsilon-v) \varepsilon-b \Delta_{D} \dot{y} \varepsilon \\
& -\left(k_{I}^{*}+a-b k_{D}^{*} k_{I}^{*}\right) v \varepsilon-\boldsymbol{h}^{T} \mathbf{z} \varepsilon \\
& -\left(1-b k_{D}^{*}\right) \tau \operatorname{sgn}[y] \varepsilon \\
& -\mathbf{z}^{T} \boldsymbol{Q} \mathbf{z}-\mathbf{z}^{T} \boldsymbol{P} \boldsymbol{g} \varepsilon+\mathbf{z}^{T} \boldsymbol{P} \boldsymbol{g} v \\
& -k_{I}^{*} v^{2}+k_{I}^{*} \varepsilon v-\Delta_{I}(\varepsilon-v) v-\tau \operatorname{sgn}[y] v \\
& -\frac{b}{\gamma} \Delta_{P} \dot{k}_{P}-\frac{1}{\gamma} \Delta_{I} \dot{k}_{I}-\frac{b}{\delta \gamma} \Delta_{D} \dot{k}_{D}
\end{aligned}
$$

Furthermore, from (6), it follows that

$$
\begin{aligned}
\dot{S} \leq & -\frac{1}{2} \alpha_{1} \varepsilon^{2}-\frac{1}{2} \alpha_{2}\|z\|^{2}-\frac{1}{2} \alpha_{3} v^{2} \\
& -\frac{b \sigma}{2 \gamma} \Delta_{P}{ }^{2}-\frac{\sigma}{2 \gamma} \Delta_{I}^{2}-\frac{b \sigma}{2 \delta \gamma} \Delta_{D}^{2}+\beta
\end{aligned}
$$


where

$$
\begin{aligned}
\alpha_{1}= & b k_{P}^{*}+b k_{D}^{*} k_{I}^{*}-3|a|-2 k_{I}^{*}-\|\boldsymbol{h}\|^{2}-\|\boldsymbol{P} \boldsymbol{g}\|^{2} \\
\alpha_{2}= & 2 \lambda_{\min }[\boldsymbol{Q}]-3 \\
\alpha_{3}= & \left(1-b k_{D}^{*} k_{I}^{*}\right) k_{I}^{*}-|a|-\|\boldsymbol{P} \boldsymbol{g}\|^{2} \\
\beta= & \frac{\tau^{2}}{2}\left(\frac{1}{b k_{P}^{*}}+b k_{D}^{*}+\frac{1}{k_{I}^{*}}\right) \\
& +\frac{b \sigma}{2 \gamma}\left(k_{P}^{*}\right)^{2}+\frac{b \sigma}{2 \gamma}\left(k_{I}^{*}\right)^{2}+\frac{b \sigma}{2 \delta \gamma}\left(k_{D}^{*}\right)^{2}
\end{aligned}
$$

Here, choose $\boldsymbol{Q}, k_{P}^{*}, k_{I}^{*}$ and $k_{D}^{*}$ so that $\alpha_{i}>0, i=1,2,3$. Then, we have

$$
\begin{aligned}
& \dot{S} \leq-\alpha S+\beta \\
& \alpha=\min \left[\frac{\alpha_{1}}{1+b k_{D}^{*}}, \frac{\alpha_{2}}{\lambda_{\min }[\boldsymbol{P}]}, \alpha_{3}, \sigma\right]
\end{aligned}
$$

which yields,

$$
S(t) \leq S(0) \exp (-\alpha t)+\frac{\beta}{\alpha}, t \in\left[0, t_{F}\right)
$$

Hence, from (9), it is verified that all the signals in the adaptive control system are bounded on $\left[0, t_{F}\right)$.

The block diagram of the proposed control system is illustrated in Fig. 1.

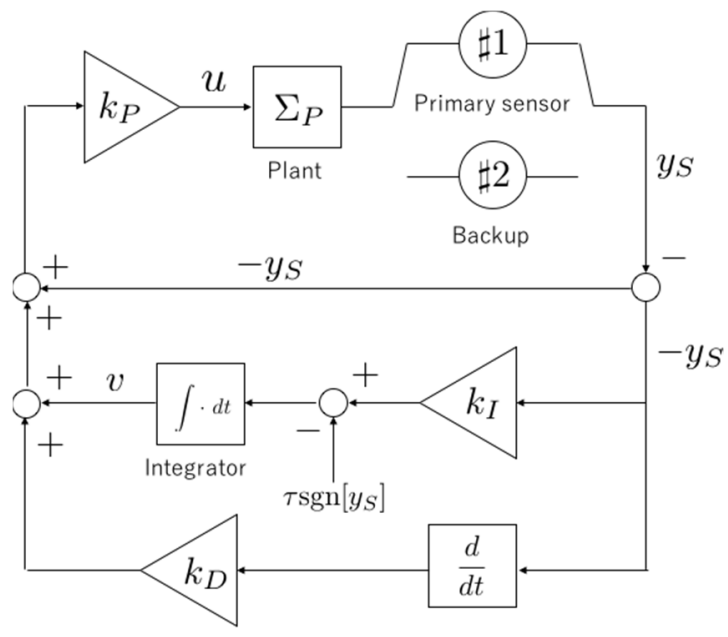

Fig. 1. Block diagram of the proposed adaptive PID control system with self-repairing function.

\section{Failure Detection \& Repairing}

This section shows the concrete detection method utilizing the integrator (5).

From the above discussion, on the time period $\left[0, t_{F}\right)$, there exists $\Gamma \in \mathbb{R}^{+}$such that

$$
|v(t)|<\Gamma, t \in\left[0, t_{F}\right)
$$

However, if the sensor fails, and $\varphi=0$, then the output of the integrator can be represented by

$$
v=v\left(t_{F}\right)-\int_{t_{F}}^{t} \tau \mathrm{dt}=v\left(t_{F}\right)-\tau\left(t-t_{F}\right)
$$

Clearly, $v$ is unbounded with respect to $t$. Hence, the inequality (14) hold no longer. By using this unstable behavior, we can find failure, and define the detection time $t_{D}$ by

$$
t_{D}:=\min \{t|| v(t) \mid \geq \Gamma\}
$$

Next, we consider the faulty situation where $\varphi \neq 0$. Then, the solution $\left(v, k_{I}\right)$ obeys

$$
\begin{aligned}
& \dot{v}=-k_{I} \varphi-\tau \operatorname{sgn}[\varphi] \\
& \dot{k}_{I}=-\sigma k_{I}-\gamma \varphi(\varphi-2 v)
\end{aligned}
$$

Hence, the equilibrium point $\left(\bar{v}, \bar{k}_{I}\right)$, is given by

$$
\begin{aligned}
& \bar{v}=\frac{\varphi}{2}-\frac{\sigma \tau}{2 \gamma \varphi^{2}} \operatorname{sgn}[\varphi] \\
& \overline{k_{I}}=-\frac{\tau}{\varphi} \operatorname{sgn}[\varphi]
\end{aligned}
$$

Now, we suppose that $\Gamma, \gamma, \sigma$ and $\tau$ are selected so that

$$
\Gamma<\left|\frac{\varphi}{2}-\frac{\sigma \tau}{2 \gamma \varphi^{2}}\right|
$$

Then, even if $v$ approaches to the equilibrium point, it hits the threshold $\Gamma$. Therefore, the detection time $t_{D}$ defined by (16) exists when $\varphi \neq 0$. Failure detection can be successfully achieved. After detection, the failed sensor is replaced with the backup, and the control stability can be maintained.

\section{Numerical Simulation}

To confirm the effectiveness of the proposed method, the numerical simulation is explored.

Consider the following unstable plant:

$$
\begin{array}{ll}
\dot{y}=y+2 u+z, & y(0)=1 \\
\dot{z}=-z+y, & z(0)=-0.5
\end{array}
$$


The failure scenario is supposed that

$$
t_{F}=25[s], \varphi=y\left(t_{F}\right)
$$

In the simulation, they are assumed to be unknown.

For the above plant, we construct the adaptive PID controller based on (4) and (5). By trial and error, the design parameters are selected as follows.

$$
\sigma=0.01, \gamma=1, \delta=0.1
$$

The other parameters for failure detection are given by

$$
\tau=1, \Gamma=1
$$

The simulation results are shown in Figs. 2 and 3.
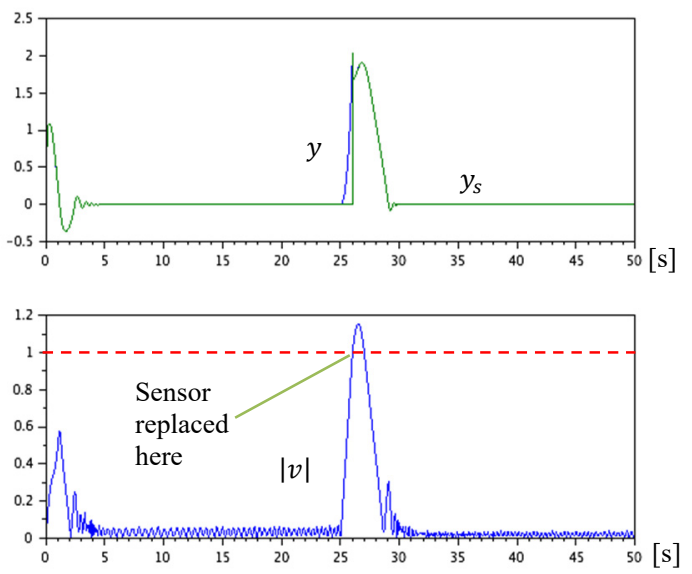

Fig. 2. Simulation results: the measured output, the actual output and the absolute value of the the integrator output.
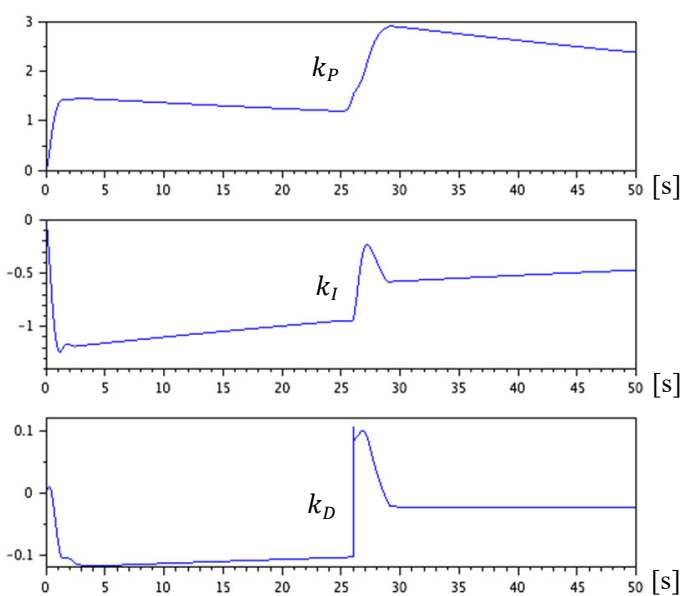

Fig. 3. Simulation results: the three adaptive PID gains.

In Fig. 2, the measured output $y_{S}$, the actual output $y$ (top) and the absolute value of $v$ (bottom) are shown.
Also, in Fig. 3, the three adaptive gains, $k_{P}$ (top), $k_{I}$ (middle) and $k_{D}$ (bottom) are shown.

From these results, it is clear that the failure (17) can be found and the failed sensor is replaced at $t_{D} \cong 26$ [s]. Furthermore, the control system can be well stabilized, and the actual output converges to a very small ball before and after the failure.

\section{Conclusions}

In this paper, a new adaptive PID control system has been developed that has self-repairing function against sensor failures, and the concrete failure detection is shown by using the integrator in the adaptive PID controller. The applications to MIMO case, nonlinear systems and so on are still left in the future works.

\section{Acknowledgment}

This work is supported by JSPS KAKENHI Grant Number JP16K06429.

\section{References}

1. R. Isermann, R. Schwarz and S. Stolzl: Fault-tolerant, Drive-by-wire systems, IEEE Control Systems Magazine, Vol. 22, No. 5, 2002, pp. 64-81.

2. Y. Zhang and J. Jiang: Biographical review on reconfigurable fault-tolerant control systems, Annual Reviews in Control, Vo. 32, No. 2, 2008, pp. 229-252.

3. K. Zhang, B. Jiang and V. Cocquempot: Fast adaptive fault estimation and accommodation for nonlinear time-varying delay systems, Asian Journal of Control, Vol. 11, No. 6, 2009, pp. 643-652.

4. Y. Wan, D. Zhou, S. J. Qin and H. Wang: Active faulttolerant control for a class of nonlinear systems with sensor faults, Int. J. of Control, Automation and Systems, Vol. 6, No. 3, 2008, pp. 339-350.

5. M. Takahashi: Self-repairing PI/PID control against sensor failures, Int. J. Innovative Computing, Information and Control, Vol. 12, No. 1, Feb. 2016, pp. 193-202. 